\title{
Young X-ray-Emitting Supernovae in Galaxies
}

\author{
Eric M. Schlegel ${ }^{1,2} \dagger$ \\ ${ }^{1}$ High Energy Astrophysics Division, Smithsonian Astrophysical Observatory, Cambridge, MA \\ 02138 USA \\ email: eschlegel@cfa.harvard.edu \\ ${ }^{2}$ Department of Physics and Astronomy, University of Texas-San Antonio, \\ San Antonio, TX 78249 USA \\ email: eric_schlegel@utsa.edu
}

\begin{abstract}
This talk reviews the observations of the high-energy emission of young supernovae, providing an update from previous reviews in 1995 and 2003. A summary plot shows the number distribution of X-ray luminosities, currently totalling 25 supernovae, from which it is clear that SN IIP are weak X-ray sources, SN IIn are very luminous sources, and SN Ib/c cover a broad range in luminosity.
\end{abstract}

Keywords. supernovae; supernovae: individual; X-rays: individual; supernova remnants.

\section{Introduction}

The X-ray emission of supernovae ( $\mathrm{SNe}$ ) provides insight into the late phases of stellar evolution by probing the explosion and subsequent interaction of the outgoing shock with the progenitor's circumstellar medium. Studies of this X-ray emission commenced with the discovery of SN1980K in NGC 6946 by Canizares et al. (1982) and accelerated with the launches of ROSAT and ASCA in the 1990s. The use of both satellites yielded studies of three important objects, SN1978K in NGC 1313, SN1993J in NGC 3031 (M81), and SN1987A (Schlegel et al. (1999), Schlegel, Petre, \& Colbert (1996), Ryder et al. (1993); Immler et al. (2001), Zimmermann et al. (1994), Suzuki \& Nomoto (1995), Kohmura et al. (1994), Leising et al. (1994); and Hasinger, Aschenbach, \& Truemper(1996) and references therein, respectively). In addition, observations of SN1998bw using BeppoSAX provided the first link between the X-ray afterglow of a GRB and a SN (Pian et al. (1999)).

The observations have been reviewed in Immler \& Lewin (2003) and Schlegel (1995). Here, I update these reviews to the current epoch (circa July 2005).

\section{Emission Mechanisms}

The emission mechanisms may be divided into two groups based on the temporal evolution.

The first group of mechanisms may be labelled as 'prompt' or 'early'. The mechanisms include Compton scattering of $\gamma$-rays, inverse Compton scattering of optical or radio photons, and the prompt thermal burst of photons associated with the break-out of the shock through the progenitor's atmosphere. Compton scattering predicts zero X-ray flux at energies below $\sim 16-20 \mathrm{keV}$ because of the photoelectric absorption. Inverse Compton scattering may produce X-rays but is expected to be confined to the earliest phases; the process is temporally limited as the expansion causes the debris field to become

$\dagger$ This research was supported by NASA Contract NAS8-39073 to SAO for the Chandra Observatory. 


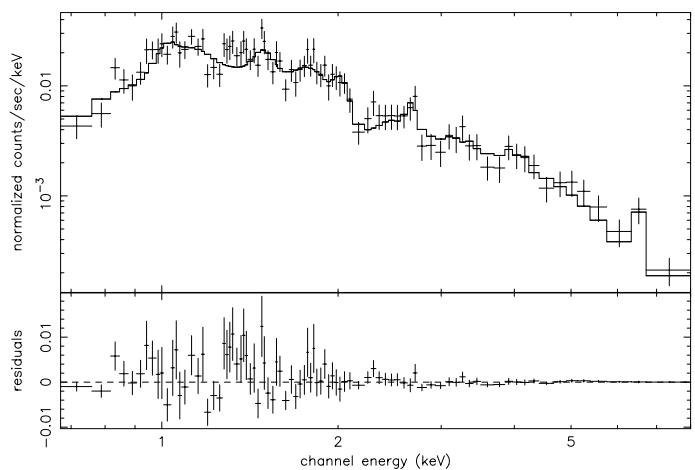

Figure 1. SN1986J as observed by XMM-Newton. A feature at $\sim 1 \mathrm{keV}$ is consistent with $\mathrm{Ne}$ emission; Fe K emission is also reported.

increasingly optically thin. The prompt thermal burst is expected to produce $\sim 100 \mathrm{keV}$ photons, but the burst should be brief, on the order of seconds. For a review, please see Schlegel (1995) and references therein.

The second group consists of the 'sustained' or 'late' mechanisms and include X-rays arising from pulsar input and circumstellar (CS) interactions. The pulsar input mechanism is expected to produce a maximum luminosity of $\sim 5 \times 10^{38} \mathrm{erg} \mathrm{s}^{-1}$ with an efficiency of $\sim 1.5 \%$ as described in Chevalier \& Fransson (1992). The CS interaction has been described by Chevalier \& Fransson (1994). In this process, the outgoing shock probes the CS medium and is expected to generate hard X-rays $(>10 \mathrm{keV})$ for the first $\sim 100$ days. As the outgoing shock plows up material, a reverse shock is eventually established that probes the progenitor 'atmosphere'. The spectrum of the reverse shock softens as the optical depth decreases. Measurements of the decline of the X-ray light curves in a soft and a hard band in principle provide measures of the density exponents of the progenitor and CS matter.

\section{Recent Observations}

Here, I provide a brief summary of observations obtained over the past few years.

SN1970G in M101 has been recovered in a subset of the 1 Msec Chandra observation of M101 and is described in Immler \& Kuntz (2005). A light curve was obtained by re-analyzing a set of ROSAT observations once the Chandra observation showed that the ROSAT sources were consistent with SN1970G. The detected decline rate of $-1.7 \pm 0.6$ is typical of the late emission of SNe.

P. Chandra and co-workers using Chandra observed SN1995N and reported in Chandra et al. (2005). An 'uptick' in the ROSAT+ASCA light curve, reported by Fox et al. (2000), was shown to be the emission of blended sources. Interpolating the trend between the ROSAT and Chandra data points at the $A S C A$ epoch provided an approximate measure of the SN emission. Re-scaling of the Chandra flux for SN1995N and convolving it plus the surrounding field with the $A S C A$ point spread function showed that the original $A S C A$ extracted flux and the convolved flux were consistent to within 20-25\%. Blended point sources at the $A S C A$ epoch provide a natural explanation for the apparent increase. The extracted Chandra spectrum of SN1995N shows evidence for enhanced Ne emission at $\sim 1 \mathrm{keV}$.

An XMM-Newton GTO observation of SN1986J in NGC 891 has been described by Temple et al. (2005) (Figure 1). The spectrum shows evidence for enhanced Ne, S, and possibly $\mathrm{Mg}$ as well as the presence of an emission line at $\mathrm{Fe} \mathrm{K} \alpha$. The slope of the light 


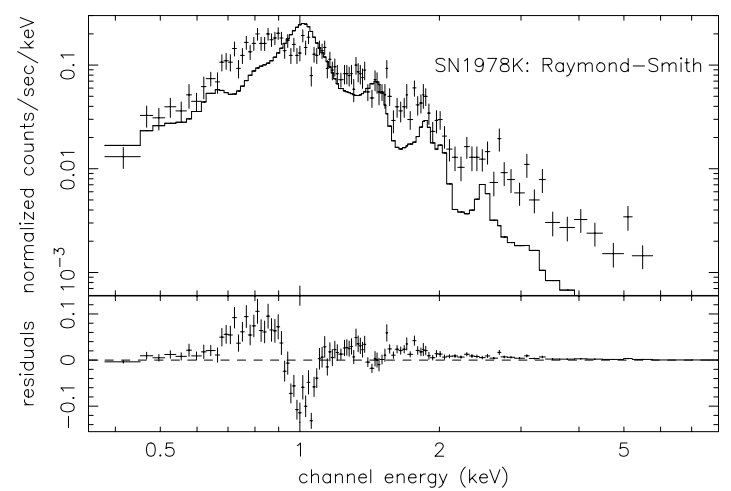

Figure 2. The spectrum from a Chandra observation of SN1978K in NGC 1313 with the best-fit $A S C A$ spectrum overlaid. Clear spectral evolution has occurred.

Supernovae by Luminosity

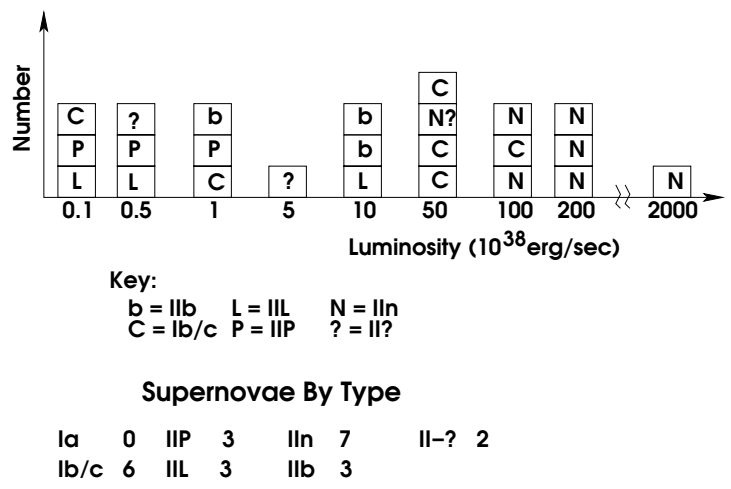

Figure 3. The distribution of X-ray emitting SNe by luminosity and type. For this figure, no adjustment has been made to generate a uniform energy band from which the flux and luminosity were calculated. As a result, the figure illustrates general trends, but may be incorrect in detail.

curve is $-2.99 \pm 0.45$ whereas free-free emission or line emission produce slopes of -1 and -1.7 , respectively. As the slope is determined by several ROSAT data points, it is possible that the point spread function of the ROSAT mirrors provides extra contamination however, the authors took care to account for the resolution. The slope thus remains a puzzle for future observations of SNe.

SN1988Z is still present in a Chandra observation as described by Schlegel \& Petre (2005). The quantile color (Hong, Schlegel, \& Grindlay (2004)) is consistent with a temperature of $\sim 2 \mathrm{keV}$. The light curve shows a decline that is slower than that implied by considering only the ROSAT data points. In contrast to SN1986J, for SN1988Z, spatial resolution is not an issue as the Chandra field shows the sources are well-separated.

Finally, Schlegel et al. (2004) report that SN1978K, the SN-equivalent of a particular well-known advertising bunny, is still generating $\sim 10^{39} \mathrm{erg} \mathrm{s}^{-1}$. Spectral evolution is definitely present as the best-fit model from the $A S C A$ epoch no longer is a good fit (Figure 2). A two-temperature model is required with soft $(0.6 \mathrm{keV})$ and hard $(3.2 \mathrm{keV})$ components. The soft band light curve remains consistent with a constant while the hard band light curve shows a drop by $\sim 50 \%$ from the $A S C A$ epoch. This likely signals the start of the decline of SN1978K. 
Table 1. Band Passes for X-ray Luminosities of SNe

\begin{tabular}{lclclc}
\hline Band & Number & Band & Number & Band & Number \\
$0.3-2$ & 2 & $0.1-2.4$ & 6 & $0.2-2$ & 1 \\
$0.5-2$ & 1 & $2-10$ & 4 & $0.5-10$ & 3 \\
$0.3-5$ & 1 & $0.2-10$ & 1 & $0.1-10$ & 1 \\
$0.4-8$ & 1 & $0.5-8$ & 2 & & \\
\hline
\end{tabular}

Note: energies are in keV; 'Number' represents \# SNe luminosities reported in that band.

New SNe have been detected, but generally have been observed only once (atrocious!) or twice (bad!) each. These include 2001em (Ib/c), 2001gd (IIb), 2001ig (IIb), 2002hh (II), 2002hi (IIn), 2003bg (Ic), 2003L (Ic), and 2004dj (IIP).

We can then update the numbers: Schlegel (1995) reviewed $5 \mathrm{SNe}(78 \mathrm{~K}, 80 \mathrm{~K}, 86 \mathrm{~J}$, 87A, 93J); Immler \& Lewin (2003) described an additional $10 \mathrm{SNe}$ (79C, 88Z, 94I, 94W, 95N, 98S, 98bw, 99em, 99gi, and 02ap). Currently, 24 SNe are present on S. Immler's list† of SNe. The number should be 25: SN1968D was recovered as described in Holt et al. (2003). This list leads to Figure 3 showing the distribution of SNe by luminosity (note that the luminosity axis is not to scale).

\section{X-ray Emission by SN Type}

\subsection{SN Ia}

Type Ia SNe have been predicted to be strong X-ray sources. Unfortunately, to date, none have been detected. The best upper limit for a SN Ia prior to the launches of Chandra and XMM-Newton was that of Schlegel \& Petre (1993) for SN1992A from a ROSAT observation obtained at an age of +15 days. The estimated limit was $1.5 \times 10^{38} \mathrm{erg} \mathrm{s}^{-1}$ in the $0.5-2 \mathrm{keV}$ band. The value was correctly criticized for not considering circumstellar absorption. It still represents a real upper limit, however, it does not provide a limit on the amount of circumstellar absorption. The newest upper limit, by Hughes et al. (2005) from a Chandra observation of SN2002bo at an age of +2 days, is $5 \times 10^{38} \mathrm{erg} \mathrm{s}^{-1}$ in the 2-8 keV band. As the host galaxy is NGC 3190 ( $\mathrm{D} \sim 19-22 \mathrm{Mpc}$ ), there remains room for improvement if Nature cooperates.

\subsection{SN IIP}

In 2001, Schlegel (2001) hypothesized that SN IIP have very little CS matter and will never be strong X-ray sources. That hypothesis was based upon Chandra observations of SN1999em and SN1999gi. Since that time, the only additional IIP object is SN2004dj (NGC 2403). The detected luminosity does not counter the hypothesis, being in the $10^{36-37}$ erg s $^{-1}$ range.

\section{3. $S N \mathrm{Ib} / \mathrm{c}$}

The association of GRBs and SN Ib/c was firmly established with the observations of GRB 030329 and SN2003dh by Stanek et al. (2003). The key question is whether every GRB produces an $\mathrm{SN} \mathrm{Ib/c}$ and vice versa. The available evidence implies the answer is 'no': Soderberg et al. (2005) argue that radio emission from a sub-relativistic jet would spread into our line-of-sight regardless of the pointing direction of the original jet. Their radio survey shows $<17 \%$ of $\mathrm{Ib} / \mathrm{c}$ SNe show radio emission, suggesting a small fraction of jets are produced. We clearly have more to learn about the association of GRBs and SNe.

$\dagger$ Available at lheawww.gsfc.nasa.gov/users/immler/supernovae_list.html 


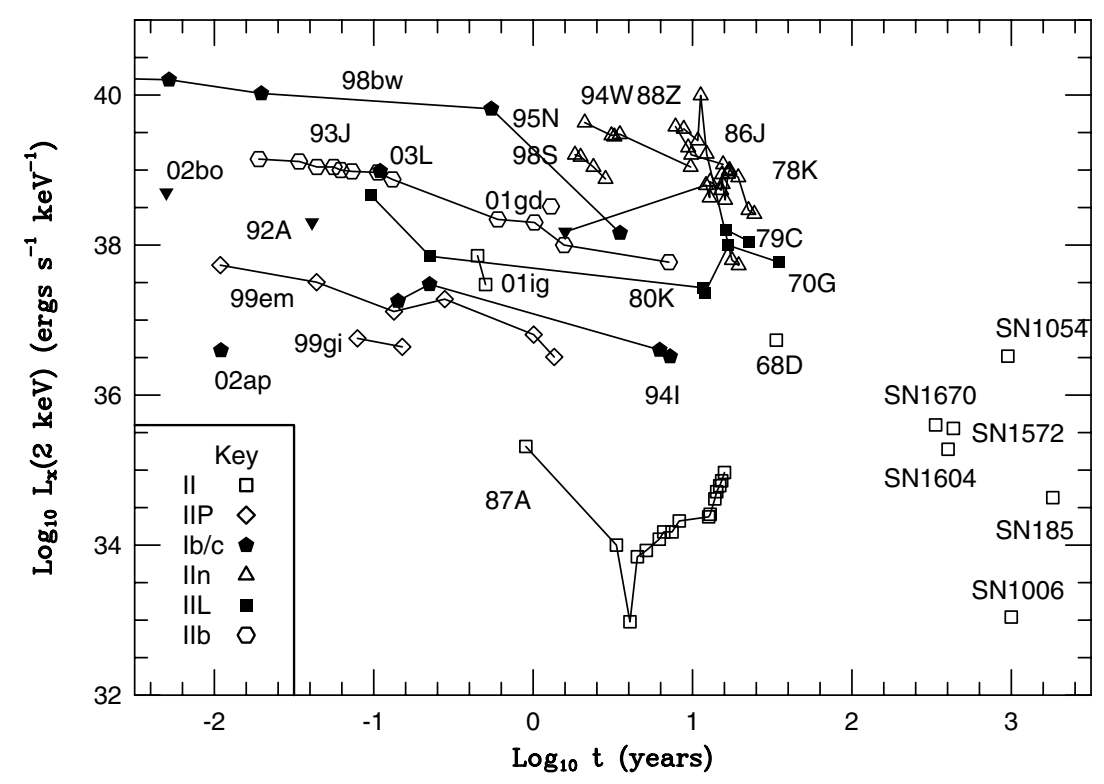

Figure 4. Plot of most of the available observations of the X-ray emission of SNe at $2 \mathrm{keV}$. The "2 keV" flux was chosen because it is common to all bandpasses of the various X-ray detectors represented. Missing from the figures are data points for 2004dj, 2003bg, 2002hi, 2002hh, and $2001 \mathrm{em}$. Of these, all are single points except $2004 \mathrm{dj}$. The key symbols apply to the $\mathrm{SNe}$ and not the remnants in the lower right corner. A variety of remnants are presented to show the eventual 'end point' of the SNe light curves.

Finally, we may aggregate all (nearly all!) of the light curve data to date by estimating the flux at $2 \mathrm{keV}$. Why $2 \mathrm{keV}$ ? To date, this energy is common to every satellite that has been used to study the X-ray emission of SNe (Table 1). Figure 4 shows the resulting light curves. The light curves have been keyed to the SN type and include the two upper limits on the SNe Ia. On the lower right side of the figure are shown the $2 \mathrm{keV}$ luminosities of several well-known SN remnants, the eventual destination of each of the light curves. From this figure, we may infer that all of the SN types are represented with detections except for SN of Type Ia, that the least luminous objects appear to be Type IIP (and highlight the distinct difference of SN1987A), that the most luminous objects are SN IIn, and that the SN Ib/c show a very broad range in luminosity (a factor of several 1000 separate SN2002ap and SN1998bw). This figure also highlights the rather spotty temporal coverage as fully $1 / 3$ of the objects are present with one or two data points, and an additional 1/3-1/2 are represented by $3-4$ data points, leaving about $1 / 5$ (ie, $\sim 4$ ) with sufficient temporal coverage that a study of trends could be possible $(78 \mathrm{~K}, 87 \mathrm{~A}$, 93J, and 99em). This illustrates the need for better temporal coverage of a range of SN types.

\section{Summary}

The X-ray emission of SNe is hard at early times, then softens. The observations to date provide a mix of slow and fast fading, suggesting that a higher rate of coverage could provide detailed information on the late stages of the progenitor's mass loss. As a 
result of the outward propagation of the shock at velocities of 1 to a few $\times 10^{4} \mathrm{~km} \mathrm{~s}^{-1}$, we view the mass loss history at a considerably increased rate.

The data collected to date also provides some observational goals. First, the observation of a nearby SN Ia $(\mathrm{D} \lesssim 5-8 \mathrm{Mpc})$ would either detect or set a critical upper limit on the presence of a circumstellar medium. To avoid possible interpretation problems, the detector should cover a hard X-ray band. Second, the detection and evolution of emission lines in a SN IIP would provide details for the hypothesized tenuous circumstellar medium surrounding these objects. Third, SN Ic and SN IIn objects, preferably more than one of each, need excellent temporal coverage to follow their light curves and spectral evolution.

\section{Acknowledgements}

I thank the conference organizers for the invitation to speak. The research and travel were supported by the Chandra X-ray Center through NASA contract number NAS839073 to the Smithsonian Astrophysical Observatory for the operation of the Chandra Observatory.

\section{References}

Canizares, C., Kriss, G., \& Feigelson, E. 1982, ApJ, 253, L17

Chandra, P., et al. 2005, ApJ, 629, 933

Chevalier, R. \& Fransson, C. 1992, ApJ, 395, 540

Chevalier, R. \& Fransson, C. 1994, ApJ, 420, 268

Fox, D. W., et al. 2000, MNRAS, 319, 1154

Hasinger, G., Aschenbach, B., \& Truemper, J. 1996, A\&A, 312, L9

Holt, S., et al. 2003, ApJ, 588, 792

Hong, J. S., Schlegel, E. M., \& J. E. Grindlay, 2004, ApJ, 614, 508

Hughes, et al. 2005, in preparation

Immler, S. \& Kuntz, K. 2005, astro-ph/0506023

Immler, S. \& Lewin, W. 2003, in Supernovae and Gamma-Ray Bursters, ed. K. Weiler (New York: Springer), 91

Immler, S., Aschenbach, B., \& Wang, Q. D. 2001, ApJ, 561, L107

Kohmura, Y., et al. 1994, PASJ, 46, L157

Leising, M. D., et al. 1994, ApJ, 431, L95

Pian, E., et al. 1999, A\&AS, 138, 463

Ryder, S., et al. 1993, ApJ, 416, 167

Schlegel, E. M. 2001, ApJ, 556, L25

Schlegel, E. M. 1995, Rprt Prog Phys, 58, 1375

Schlegel, E. M. \& Petre, R. 2005, in preparation

Schlegel, E. M., et al. 2004, ApJ, 603, 644

Schlegel, E. M., et al. 1999, AJ, 118, 2689

Schlegel, E. M., Petre, R., \& Colbert, E. J. M. 1996, ApJ, 456, 187

Schlegel, E. M. \& Petre, R. 1993, ApJ, 412, L29

Soderberg, A., et al. 2005, astro-ph/0507147

Stanek, K., et al. 2003, ApJ, 591, L17

Suzuki, T. \& Nomoto, K. 1995, ApJ, 455, 658

Temple, R., Raychaudhury, S., \& Stevens, I. 2005, astro-ph/0506657

Zimmermann, H.-U., et al. 1994, Nature, 367, 621 


\section{Discussion}

ZEZAS: The possibility that some ULXs could be SNRs is valid if we have single snapshot observation. However, for many galaxies we now have multiple exposures showing that many ULXs are variable associating them with X-ray binaries.

SCHLEGEL: I agree.

ERACleOus: Can you please summarize the best estimate of the duration of the "early hard phase" and the best estimate of the $2-10 \mathrm{KeV}$ luminosity during this phase.

SCHLEGEL: SN 1993 is probably the best case for answering this question. The duration is about 100 days and luminosity is about $10^{39} \mathrm{erg} / \mathrm{s}$, although theoretically it could reach $10^{40} \mathrm{erg} / \mathrm{s}$. 